\title{
Análisis comparativo de alternativas viales entre Manizales y Mariquita (Colombia) a través de un estudio de accesibilidad territorial
}

\author{
Cristian M. Loaiza, Juan M. Holguín y Diego A. Escobar \\ Facultad de Ingeniería y Arquitectura, Dpto. de Ingeniería Civil, Universidad Nacional de Colombia - Sede \\ Manizales. Cra 27 \# 64-60 Manizales, Caldas - Colombia. (e-mail: cmloaizaa@unal.edu.co, \\ jmholguinc@unal.edu.co, daescobarga@unal.edu.co)
}

Recibido Feb. 9, 2016; Aceptado Abr. 13, 2016; Versión final Abr. 26, 2016, Publicado Oct. 2016

\begin{abstract}
Resumen
En este artículo se analiza la conexión vial de la ciudad de Manizales (Caldas) con el municipio de Mariquita (Tolima), trayecto que posee una topografía abrupta. Por ello, es que el diseño geométrico de la vía actual posee unos radios de curvaturas mínimos, además de tener entretangencias muy cortas, haciendo incómodo el viaje para el usuario. Para corregir el problema anterior se plantearon cuatro alternativas viales, tres propuestas por el Instituto Nacional de Vías y una por una Asociación Publico Privada. Ninguna de ellas tomó en cuenta el análisis de accesibilidad de los territorios que están en el área de influencia a esas infraestructuras planteadas. La metodología aplicada es un análisis de accesibilidad que arroja unas curvas isócronas y curvas de porcentaje de ahorro de tiempo medio de viaje. Posteriormente se cruzan con variables demográficas y económicas, obteniendo como resultado que la mejor alternativa es aquella en la que el trazado vial cubre más población y mayor producto interno bruto de la región, constituyéndose en una solución que beneficia en un mayor porcentaje a los habitantes de la zona analizada.
\end{abstract}

\section{Comparative analysis of road alternatives between Manizales and Mariquita (Colombia) trough of study of regional accessibility}

\begin{abstract}
This paper analyzes road connection of the city of Manizales (Caldas) with the municipality of Mariquita (Tolima) in Colombia. This route has steep topography, causing an inappropriate geometric design of the current road, such as minimum curvature radius and limited length between curves, making the trip uncomfortable for users. To solve the above problem were proposed four road alternatives, three made by the National Institute of Roads and one by a Public Private Partnership. None of them considered the geographic accessibility of the territories that belong to the area of influence. The methodology applied is an analysis of accessibility, which has as result the isochrones curves. After that, the economic and demographic variables are crossed obtaining as a result that the best alternative is that in which the road design includes more population and higher gross domestic product of the region, being then a solution that benefits to a higher number of people of the region studied.
\end{abstract}

Keywords: territorial accessibility; road connection; average global accessibility; isochronous curves 


\section{INTRODUCCIÓN}

El rezago en infraestructura de transporte en Colombia es bastante considerable, situación que reduce las oportunidades de globalización del país, resaltando la poca conectividad transversal que actualmente existe (Duque, 2009). Colombia, en la búsqueda de una economía cada vez más sólida, ha promovido su desarrollo a través de la firma de variados tratados de libre comercio (TLC) y ante la inminente firma de un acuerdo de paz. Dado lo anterior, se pretende mejorar la competitividad del país a través del fortalecimiento de la infraestructura vial con características apropiadas que permitan una conexión eficiente entre los centros de producción y los centros de consumo y/o embarque. La conexión Manizales-Mariquita se convierte en una vía transversal valiosa para el país, considerándole una alternativa fundamental frente al corredor principal que posee el territorio nacional para conectar los centros de producción con la capital del país, adquiriendo una importante relevancia para los departamentos de Caldas y Tolima (INVIAS, 2011).

En el estudio de Sánchez (2012) se define la palabra accesibilidad como "la oportunidad relativa de interacción y contacto" pero que en la práctica se considera comúnmente como la menor o mayor facilidad para acceder de un sitio a otro, coincidiendo con Escobar et al. (2013), quienes explican que la accesibilidad se encuentra relacionada con la variable distancia, y más estrechamente con la distancia a las infraestructuras de transporte y de la forma como éstas reducen los tiempos de conexión entre dos puntos específicos; es de esta forma que los modos de transporte son los que toman protagonismo para el análisis, y de la forma como es posible involucrar esta metodología en la planificación del transporte (Litman, 2011). Según Geurs et al.(2012), los análisis de accesibilidad regional o territorial están ganando impulso para entender las actividades humanas en las respectivas zonas donde además se involucran asuntos económicos e inversiones en infraestructuras relacionadas con el transporte. Es por ello que este tipo de análisis es un apoyo técnico que muestra un panorama general de las condiciones de accesibilidad ofrecidas por infraestructuras del transporte a una región, que en cualquier momento puede apoyar la toma de decisiones respecto a modificaciones que se deseen realizar a la red vial o a los sistemas de transporte que le usan y sobre todo para establecer en qué áreas se deben aunar esfuerzos para ofrecer una mejor accesibilidad y aumentar la calidad de vida de los pobladores (Escobar y García, 2012).

Gutiérrez et al. (1994) coincide con Escobar et al. (2013), Geurs et al. (2015) y Wang et al. (2015) en el enfoque general de la accesibilidad cuando menciona que las infraestructuras de transporte constituyen un elemento clave en las políticas de desarrollo territorial donde las nuevas inversiones pueden resolver problemas de congestión o de demoras al transportar un producto de un lugar a otro, pero que también se pueden orientar a ofrecer nuevas potencialidades a aquellos espacios menos desarrollados. Gutiérrez et al (1994) citan a Biehl (1986) para señalar cuatro factores determinantes en el desarrollo regional: Las infraestructuras, la localización, la aglomeración y estructura de asentamientos y, finalmente, la estructura económica sectorial; no obstante, en el momento de evaluar alternativas de intervención en infraestructura, se deben considerar modelos territoriales de ciudad-región con el fin de aplicar conceptos sustentables y desarrollos equitativos (Venegas y Rojas, 2009). La infraestructura vial que actualmente conecta las ciudades de Manizales y Bogotá D.C. atraviesa la cordillera central, la cual se caracteriza por tener una topografía abrupta, especialmente en el tramo entre Manizales (391,640 hab.) y Mariquita (32,933 hab.). Ambas poblaciones influyen de forma directa la economía del sur oriente de Caldas y el norte del Tolima, lo cual hace que cualquier tipo de mejora o de nueva inversión sobre la infraestructura del transporte en dicho recorrido, seguramente impactarán el crecimiento de la economía regional (Rietveld y Bruinsma, 1998), un ejemplo tangible del impacto se observaría en un incremento en los precios de la tierra y las viviendas según los niveles de accesibilidad obtenidos (Du y Mulley, 2006; Martínez y Viegas, 2009).

En este trabajo se parte del estudio que realizó la firma Civiltec Ingenieros Ltda (2013) para el Instituto Nacional de Vías, donde propone tres alternativas para conectar las poblaciones de Manizales y Mariquita recorriendo gran parte de la cordillera central, buscando disminuir los tiempos de viaje y conectarse con futuros proyectos de gran relevancia para el país. Además, a las alternativas finales que el mencionado estudio revela, se evalúa también la vía que actualmente existe entre Manizales y Cambao tramo La Esperanza-Armero-Mariquita, la cual no se encuentra en óptimas condiciones por lo que se intervendrá mediante una Asociación Publico Privada (APP) para su rehabilitación y mejoramiento (Agencia Nacional de Infraestructura, 2015).

Para la investigación que se aborda se define como área de influencia directa para los cálculos los departamentos de Antioquia, Boyacá, Caldas, Chocó, Cundinamarca, Quindío, Risaralda, Santander, Tolima y Valle del Cauca, así como de la ciudad de Bogotá (figura 1). Según lo planteado por el INVIAS, la vía actual consta de $108 \mathrm{~km}$ con un tiempo de recorrido de 3.1 horas y una velocidad promedio de diseño de 35 $\mathrm{km} / \mathrm{h}$ mientras que las 4 alternativas planteadas cuentan con las características que se observan en la Tabla 1 y su ubicación general se observa en la figura 2 . 


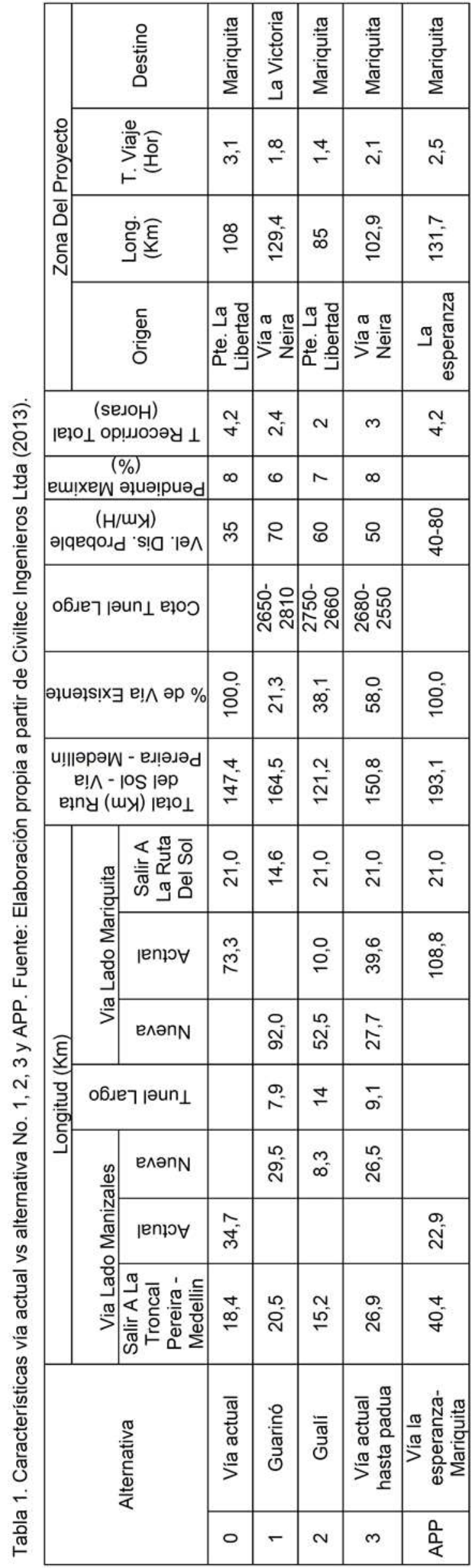
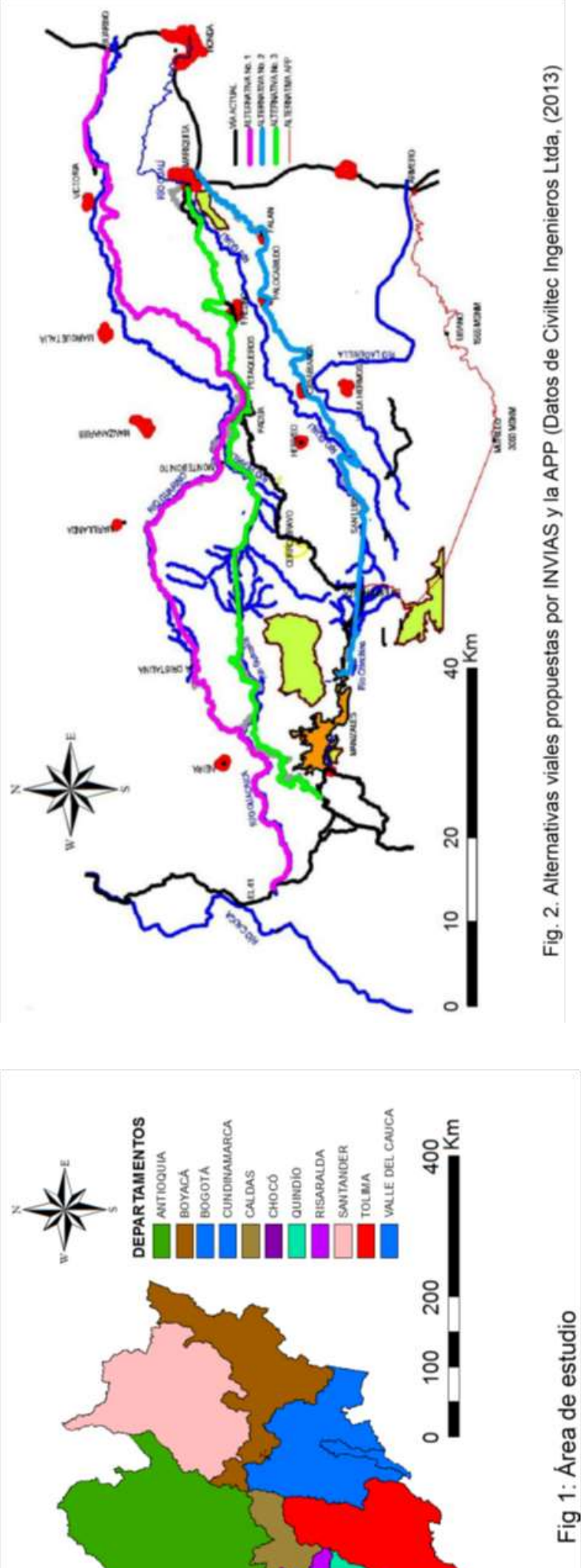
Como se ha dicho, esta zona de estudio es vital para el desarrollo socioeconómico del país, ya que integra 10 de los 32 departamentos de Colombia, que, en área superficial, población y Producto Interno Bruto corresponden respectivamente al $21.64 \%, 60.95 \%$ y al $70.92 \%$ de todo el territorio nacional, además la red vial primaria analizada corresponde aproximadamente al $39 \%$ de toda la red primaria nacional sin incluir la longitud de cada una de las alternativas analizadas. Las tres alternativas propuestas por CIVILTEC más la APP (ver Figura 2) se evalúan mediante un análisis de accesibilidad territorial, metodología que a pesar de ser muy nueva en Colombia, en otros contextos mundiales se han presentado sus bondades, como es el caso del proyecto de TERN (Trans-European road network) que fue evaluado por Gutiérrez y Urbano (1996) y la relación con el desarrollo económico (Vickerman et al., 1999).

El análisis de accesibilidad territorial es un concepto global que como su nombre lo indica se aplica a diferentes regiones del mundo para analizar problemas y planteamientos propios de una zona determinada. Un ejemplo de ello se observa en la investigación de Sánchez (2012), donde realiza un análisis de accesibilidad a la comunidad autónoma de Castilla - La Mancha (España) evaluando el impacto que las infraestructuras del transporte (carreteras y ferroviaria) imprimen en dicha región, encontrando que en las áreas de carácter intermodal y las zonas que tienen buena conexión vial se localiza casi un $90 \%$ de la población total de la región que corresponde al $50 \%$ de los municipios. Otro ejemplo de este tipo de análisis fue el realizado para Finlandia (Kotavaara et al, 2011), en el cual evaluaron el impacto que tuvieron las infraestructuras del transporte en relación con los cambios poblacionales en el período comprendido entre 1970 y 2007, encontrando que la población se fue concentrando en áreas que referían altos índices de accesibilidad, sobre todo después de la apertura económica de los 90'. Así mismo, existen investigaciones que miden los efectos de inversión en infraestructuras del transporte en relación con la cohesión regional (López et al, 2008) teniendo en cuenta indicadores económicos, en este caso, por ejemplo, se midieron los impactos que generaron las inversiones en transporte realizadas en el período 1992 - 2004 en España.

\section{METODOLOGÍA}

La metodología aplicada en esta investigación se compone de seis (6) etapas (Ver Figura 3): a) Definición de características topológicas y operativas de la red de infraestructuras del transporte actual (Alternativa 0); b) Ajuste de la red de infraestructuras del transporte actual con las características topológicas y operativas de cada una de las alternativas a estudiar (Alternativa 1 a 3 y APP); c) Recopilación de información secundaria; d) Cálculo de la Accesibilidad Media Global y Gradientes de accesibilidad para cada alternativa; e) Análisis de Cobertura espacial; y f) Análisis comparativo de resultados de cobertura espacial.

a) Definición de características topológicas y operativas de la red de infraestructuras del transporte actual (Alternativa 0): En esta etapa se tomó como información primaria la red de infraestructuras del transporte (Red vial primaria y secundaria) de los departamentos de Antioquia, Boyacá, Caldas, Chocó, Cundinamarca, Quindío, Risaralda, Santander, Tolima y Valle del Cauca, así como de la ciudad de Bogotá D.C. (Ver Figura 1). Para la definición topológica y operativa de la red de análisis (grafo), se tomó como base la información publicada de forma oficial por parte del Instituto Nacional de Vías - INVIAS. El grafo de estudio, compuesto por 31,000 nodos y 32,000 arcos, se usó para el cálculo de la Accesibilidad Media Global a partir de datos de velocidad de operación funcional (según la categoría de la vía), mediante el cálculo de caminos mínimos (tramos más cortos) entre cada par de nodos.

b) Ajuste de la red de infraestructuras del transporte actual con las características topológicas y operativas de cada una de las alternativas a estudiar (Alternativa 1 a 3 y APP): A partir de la red de infraestructuras definida en la etapa 1, se ajustan las nuevas redes de estudio según las cuatro alternativas a estudiar.

c) Recopilación de información secundaria: En esta etapa se recopiló la información secundaria necesaria para el análisis de accesibilidad territorial, como: población, PIB, actividades económicas, áreas, entre otras. Los datos socioeconómicos y demográficos del área de estudio son los publicados de forma oficial por el Departamento Administrativo Nacional de Estadística (2005), son de origen censal, público y de alcance municipal, departamental o nacional.

d) Cálculo de la Accesibilidad Media Global y Gradientes de accesibilidad para cada alternativa: En esta etapa se calcula la Accesibilidad Media Global ofrecida por la red de infraestructuras de transporte actual y de cada una de las alternativas; así mismo, se realizan los cálculos de gradiente de accesibilidad que se tendrían al comparar cada una de las alternativas con la situación actual. Para todo el proceso de cálculo se usaron los módulos Network Analyst y Spatial Analyst del software ArcGis 10.2.1.

e) Análisis de Cobertura espacial: En esta etapa se relacionan las curvas isócronas generadas en el análisis de accesibilidad media global con los datos sociodemográficos y socioeconómicos del área de estudio. Se calcula la cobertura espacial para la situación actual, cada una de las alternativas y los gradientes de accesibilidad obtenidos. 
a) Definición de características topológicas y operativas de la red de infraestructuras del transporte actual (Alternativa 0 )

b) Ajuste de la red de infraestructuras del transporte actual con las caracteristicas topológicas

y operativas de cada una de las alternativas a estudiar (Alternativa 1 a 3 y APP)

c) Recopilación de información secundaria

d) Cálculo de la Accesibilidad Media Global y

Gradientes de accesibilidad para cada alternativa

e) Análisis de Cobertura espacial

f) Análisis comparativo de resultados de cobertura espacial

Resultados,

Discusión y

Conclusiones

Fig. 3: Metodología aplicada

f) Análisis comparativo de resultados de cobertura espacial: En esta etapa se comparan los resultados de cobertura espacial obtenidos en cada una de las alternativas estudiadas, tanto desde el punto de vista de Accesibilidad Media Global como desde el punto de vista de gradiente de accesibilidad. Esta comparación permite definir cuál de las alternativas analizadas tendría mayor impacto sobre el área de estudio.

\section{FORMULACIÓN DEL MODELO}

Para el cálculo de la accesibilidad territorial ofrecida por la red de infraestructuras del transporte (red vial primaria y secundaria) del área de influencia directa de las alternativas y con fines de modelación, se toman como valores de velocidad operacional los valores funcionales de la misma según la categoría de la vía (Burns y Inglis, 2007), lo cual permitió calcular los tiempos medios de viaje sobre la red; no obstante existen recientes investigaciones de accesibilidad que toman velocidades reales de los vehículos (Li et al., 2011). El modelo que a continuación se formula se basa en la obtención del vector de tiempo medio de viaje (Tavg, Ver expresión (1)), que representa el tiempo de viaje promedio del nodo i al resto de nodos de la red Shen (2002), el cual se ajusta en esta investigación para los cálculos posteriores de cobertura espacial. Este indicador tiende a favorecer los nodos ubicados hacia el centro de la red, ya que los tiempos de viaje de aquellos nodos a todos los demás son más cortos, debido precisamente a su ubicación geográfica.

Denotando $i, j \in I, i=\{1,2, \ldots\}, j=\{1,2, \ldots\}$ como el conjunto de nodos que conforman un arco. $D_{i j}=$ distancia mínima entre un nodo $i$ y un nodo $j$ en la red de transporte. $T_{i j}=$ valor de la función objetivo. La función objetivo es minimizar el tiempo medio de viaje $\left(T_{i j}\right)$ entre un nodo i y los demás nodos j.

$T_{\text {avg }}=\frac{\sum_{j=1}^{m} t_{v i}}{(n-1)}, \quad$ siendo $i=1,2,3, \ldots, n ; j=1,2,3, \ldots, m$.

Donde, $t_{v i}=$ tiempo de viaje mínimo promedio entre el nodo i y los demás nodos del grafo; $\mathrm{n}=$ número total de nodos del grafo.

Es de notar que la mayor impedancia es entonces la velocidad de operación sobre cada arco y no la distancia entre cada par de nodos; el modelo asume que todos los nodos podrán conectarse entre sí mediante el uso de la red de infraestructuras de transporte existente. El vector de Tiempos promedio mínimos de Viaje está representado por una matriz de tamaño $n \times 1$, la cual se relaciona con las coordenadas (longitud y latitud) de cada nodo de la red generándose una nueva matriz de tamaño nX3. Es a partir de esta matriz que es posible calcular las curvas isócronas para el análisis de la accesibilidad media global. La Accesibilidad Media Global permite identificar de forma general cuáles son las zonas más accesibiles de una región, en términos de tiempo medio de viaje, dadas las características operativas de la infraestructura del tranporte que existe en una región o área de influencia determinada; un ejemplo de aplicación es el conocer que sector de una región refiere los menores tiempos medios de viaje para llegar a éste, entendiendo que dada esta condición en particular, dicho sector definiría un área de importancia estratégica para la misma región como tal. El modelo geoestadístico Kriging ordinario con semivariograma lineal fue el escogido para la predicción de la variable $T_{\text {avg }}$ a lo largo y ancho de la región de estudio, a partir del cálculo de la desviación estándar de los nodos (ecn. (2) y la varianza del conjunto de nodos (ecn. (3)). 
Desviación estándar del conjunto de nodos $T_{s d}$ :

$T_{s d}=\sqrt{\left[\sum_{i} \sum_{j}\left(D_{i j}-D_{a v g}\right)^{2}\right] /(n-1)}$,

Varianza del conjunto de nodos, a partir del cual se construye el semivariograma $T_{\text {var }}$ :

$T_{\text {var }}=\frac{\left[\sum_{i} \sum_{j}\left(D_{i j}-D_{a v g}\right)^{2}\right]}{n-1}$,

Finalmente, mediante el uso del SIG, y con la información sociodemográfica de la región en estudio, $247,067 \mathrm{Km}^{2}$ (22\% del total del país) que alberga a 29'378,099 habitantes (61\% del total del país), que producen el $71 \%$ del Producto Interno Bruto del país; se estimó el porcentaje de población, área y PIB, que se encontraban cubiertos por las curvas de tiempo medio de viaje obtenidas.

Se utilizó esta metodología para analizar las tres alternativas y la alternativa de la APP, así como para calcular las curvas gradiente obtenidas al comparar la situación que se tendría al insertar cierta alternativa en relación con la situación actual; lo anterior permite establecer, desde una misma base de análisis, cual de las alternativas refiere los mayores impactos en términos ded reducción del tiempo medio de viaje y en términos de cobertura de las tres variables analizadas (población, área y PIB). Es de resaltar que la metodología aplicada toma las velocidades funcionales de los arcos que componen la red de infraestructuras del transporte, lo cual se mitifica como un efecto simplificador (valores agragados) en relación con la aplicación del modelo propuesto, ya que se está homogenizando la velocidad funcional de todos los modos de transporte en un solo valor; dicha situación debe ser cosiderada al momento de tomar los resultados del presente modelo como base en futuros procesos de planificación regional.

\section{RESULTADOS Y DISCUSIÓN}

Los resultados y la discusión de ellos se organizan en tres sub-secciones: i) la Accesibilidad Media Global ofrecida por la red vial actual y cada una de las alternativas; ii) el Gradiente del tiempo medio de viaje donde se refleja los porcentajes de ahorro de cada una de las alternativas con respecto al escenario actual; y iii) Comparativo de los resultados de cobertura geoespacial en relación a los tiempos de viaje promedio y las variables población y Producto Interno Bruto (PIB)

\section{Accesibilidad Media Global}

En la Figura 4 se presentan los tiempos medios de viaje, en horas, para la red actual y cada una de las alternativas con que cuenta la zona de estudio, donde podemos observar que los sitios más accesibles, en contexto con la región, se encuentran ubicados en el centro de la misma. Es por ello que el eje cafetero (Caldas, Risaralda y Quindío) es el que refiere menores tiempos de viaje tanto en el escenario actual como en cada una de las alternativas que se plantean en este análisis. Confirmando lo anteriormente dicho el menor tiempo de viaje promedio ponderado por el área de los departamentos es Caldas seguido por Quindío y Risaralda, donde Caldas oscila en 6.5 horas en cada uno de las alternativas mientras que los otros dos departamentos fluctúan alrededor de 6.7 horas y 6.8 horas respectivamente. Sin embargo cabe resaltar el tiempo de viaje promedio ponderado de 8.5 horas aproximadamente en cada uno de los escenarios del departamento del Tolima debido a su posición de acuerdo a las alternativas, teniendo una diferencia considerable con los departamentos pertenecientes al eje cafetero.

Otro de los puntos importantes a considerar es el departamento del Chocó, el cual en la actualidad cuenta con $611 \mathrm{~km}$ de vías terrestres que corresponde al 4\% de la red vial de Colombia (Alcaldía de Quibdó, 2015), esta infraestrucutura se encuentra en malas condiciones y sólo conectan a su capital Quibdó y los municipios del sureste con los departamentos de Risaralda y Antioquia. Lo anterior se refleja en la baja accesibilidad de este estado siendo el peor de la región de estudio, aunque la baja presencia del gobierno y la baja fortaleza que tienen las instituciones en esta zona del país influyen en la poca inversión en infraestructura, esta afirmación se apoya en la dirección que han tomado cerca de 12,000 millones de dólares hacia este rubro en el proyecto denominado por el gobierno vías $4 \mathrm{G}$, en donde la primera ola de vías 4G se encuentran 10 proyectos los cuales tienen un costo de 5,500 millones de dólares, en la segunda ola de vías $4 G$ hay otros 9 proyectos con un costo aproximado de 6,500 millones de dólares (Agencia Nacional de Infraestructura, 2014), de estas 19 megaobras 9 se presentan en la zona de estudio con valor aproximado de 5,300 millones de dólares representando el $44 \%$ del presupuesto destinado para esta infraestructura, recordando que el área de estudio corresponde aproximadamente al $22 \%$ del territorio colombiano, por consiguiente el gobierno está tratando de ser equitativo, sin embargo se está quedando bastante corto, ejemplo de ello es el departamento del Chocó el cual sólo se ve influenciado por ciertos proyectos en los límites con el departamento de Antioquia. 

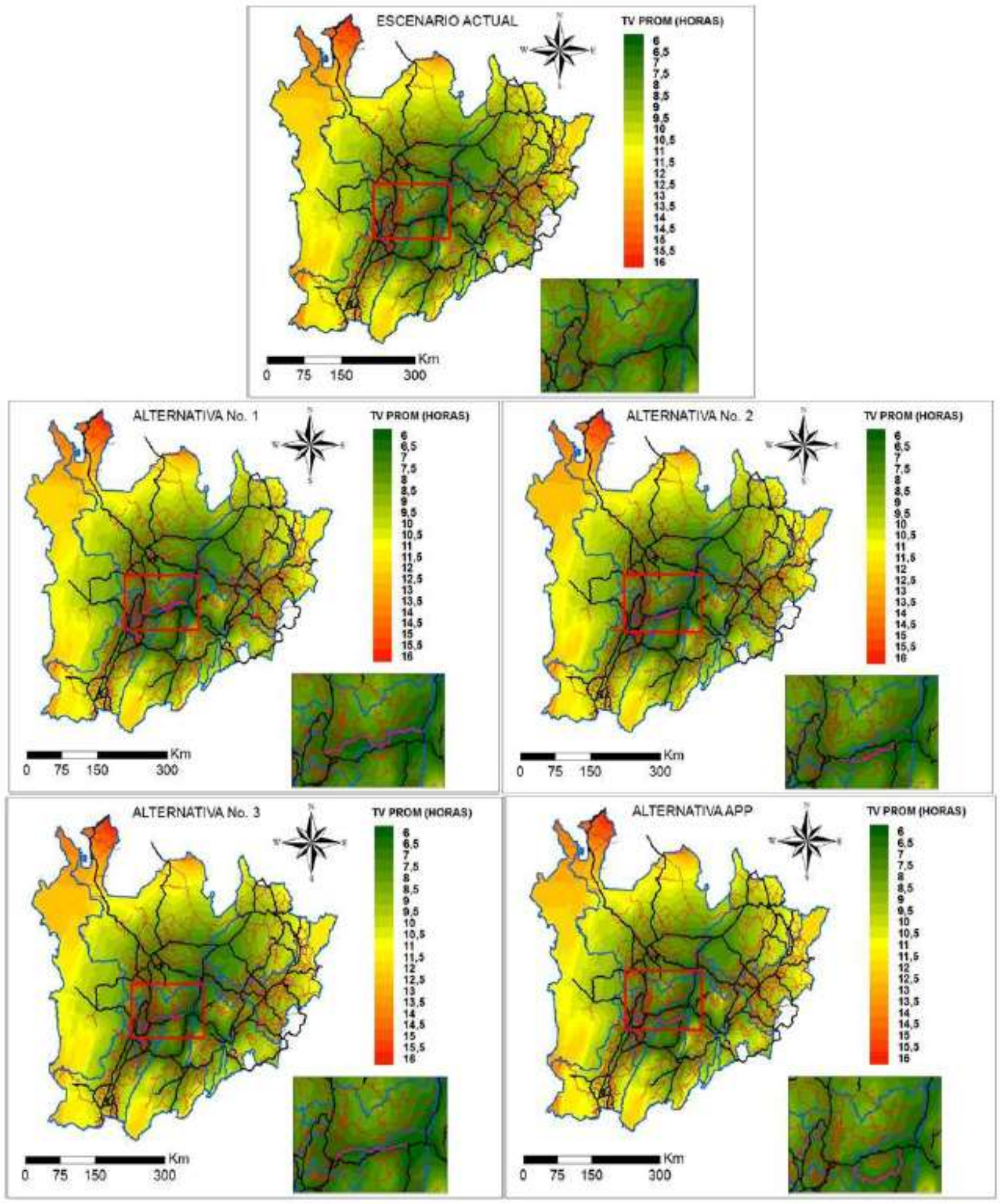

Fig. 4: Accesibilidad Media Global del escenario actual y de cada una de las alternativas

Los departamentos y municipios con mejor accesibilidad de la región analizada se muestran en la Tabla 2 , en la cual se puede observar, por ejemplo, que para el escenario actual el departamento con mejor accesibilidad media global es Caldas con tiempos medios de viaje de 7.34 horas, además tenemos que el municipio que sobresale en el departamento de Caldas con tiempos de viaje promedio de 6.64 horas es La Dorada, lo que le convierte en un territorio muy accesible en contexto con el departamento y con la región. Ahora bien de todos los municipios que integran la región de estudio, el que presenta la mejor accesibilidad media global es Honda con tiempo de viaje promedio de 6.2 horas; y al realizar el análisis de cada una de las alternativas vemos como se mejoran los tiempos de viaje promedio y como, dependiendo del trazado vial que se presente se ven mas beneficiados unos departamentos y/o municipios que otros. 
Tabla 2: Departamentos y municipios con mejor accesibilidad

\begin{tabular}{|c|c|c|c|c|c|c|}
\hline \multirow{2}{*}{ Alt. } & \multicolumn{5}{|c|}{ Departamentos Y Municipios Con Mejor Accesibilidad Media Global } \\
\cline { 2 - 7 } & Departamento & $\begin{array}{c}\text { Tv_Prom } \\
\text { (Horas) }\end{array}$ & $\begin{array}{c}\text { Municipio del } \\
\text { Departamento }\end{array}$ & $\begin{array}{c}\text { Tv_Prom } \\
\text { (Horas) }\end{array}$ & $\begin{array}{c}\text { Municipio de } \\
\text { La Region }\end{array}$ & $\begin{array}{c}\text { Tv_Prom } \\
\text { (Horas) }\end{array}$ \\
\hline Actual & Caldas & 7,34 & La Dorada & 6,64 & Honda & 6,2 \\
\hline Alt 1 & Caldas & 6,97 & Neira & 6,34 & Mariquita & 6 \\
\hline Alt 2 & Caldas & 7,19 & La Dorada & 6,46 & Honda & 6,08 \\
\hline Alt 3 & Caldas & 7,03 & Manizales & 6,28 & Honda & 6,06 \\
\hline Alt App & Caldas & 7,33 & La Dorada & 6,64 & Honda & 6,14 \\
\hline
\end{tabular}

\section{Gradiente del tiempo medio de viaje}

En la Figura 5 se presentan los gradientes de tiempos medios de viaje, en porcentaje de ahorro, comparando la red actual con cada una de las alternativas con que cuenta el área de estudio, en donde se aprecia que las zonas con mayores porcentajes de ahorro se ubican en los sectores aledaños del trazado vial de cada alternativa, sin embargo cuando se refiere a los porcentajes de ahorro promedio ponderado por el área de los departamentos se encuentra que Risaralda es el primero o segundo más favorecido por las alternativas planteadas por el Instituto Nacional de Vías, mientras que en la APP no se encuentra tan privilegiada aunque 9 de los 10 departamentos en estudio oscilan en $1 \%$ de ahorro siendo el departamento del Tolima el único que alcanza valores cercanos al 1.5\% de ahorro del tiempo de viaje en esta alternativa.

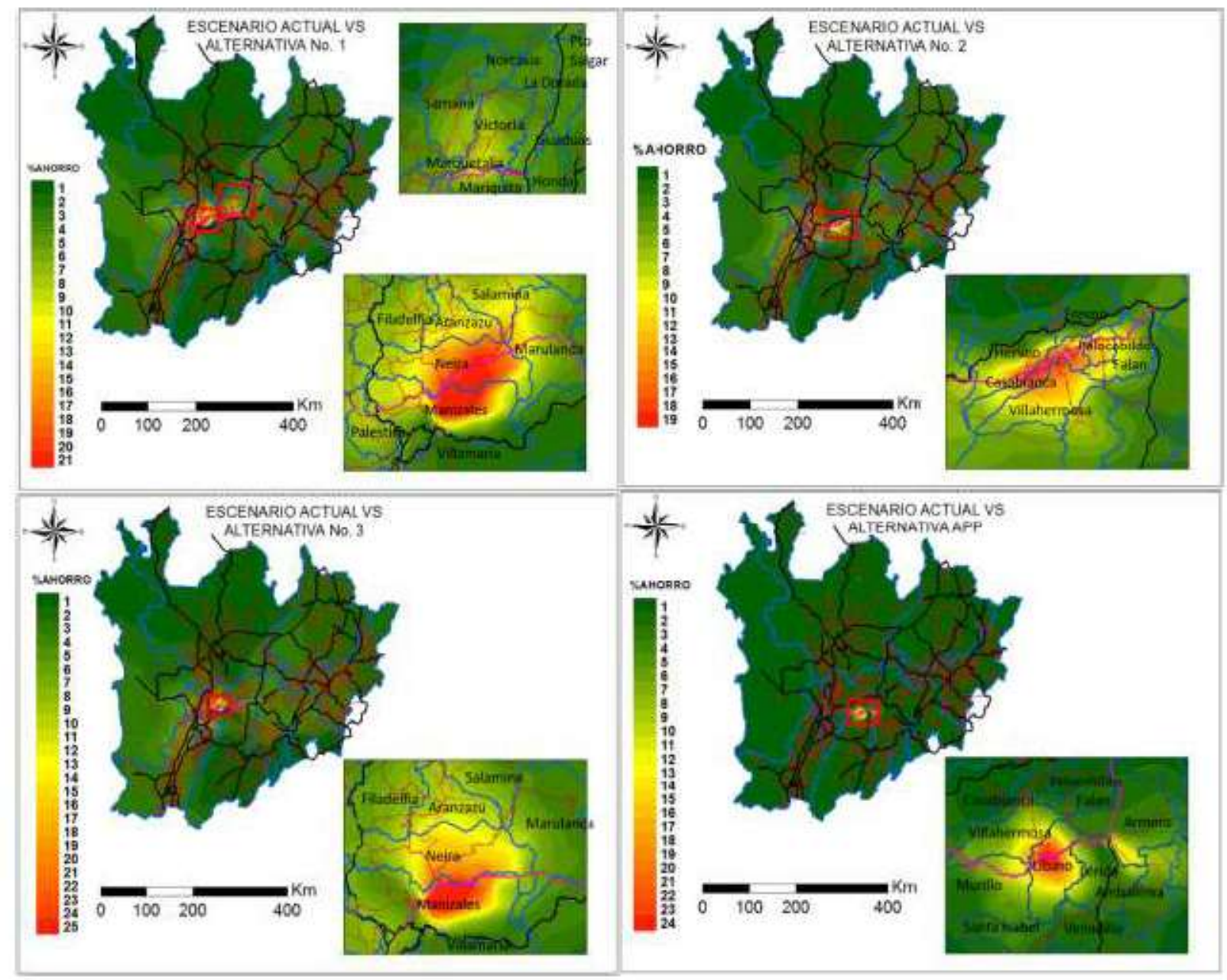

Fig. 5: Gradiente de tiempos medio de viaje (porcentjaes de ahorro) del escenario actual con cada una de las alternativas 
Es relevante abordar el departamento de Antioquia ya que es el segundo territorio de Colombia detrás de Bogotá en influencia del PIB del país, debido a que en este estudio es el que menos porcentaje de ahorro del tiempo medio de viaje presenta con valores que fluctúan cerca del 1\%. Esto se debe a que la conexión principal de este departamento con Caldas se encuentra sobre el occidente a nivel longitudinal, mientras que la infraestructura en análisis se desarrolla transversalmente y adicionalmente es sobre el oriente de Caldas.

Los departamentos y municipios con más alto porcentaje de ahorro de la región analizada se muestran en la Tabla 3, donde se tiene que para el escenario actual versus la alternativa No. 3, el departamento que presenta mayores pocentajes de ahorro con la infraestructura vial a realizar es Risaralda, con $5.74 \%$ de ahorro y como el municipio mas beneficiado de este departamento es Dosquebradas con un ahorro de $7 \%$, además, teniendo en cuenta a Caldas como referente, se tiene que el municipio con mayor ahorro es Neira con $12.25 \%$, donde sorpresivamente también es el municipio de toda la región analizada con el mayor porcentaje de ahorro. Al realizar el análisis de cada una de las alternativas vemos como existen unos pocentajes de ahorro en el tiempo de viaje promedio, y como, dependiendo del trazado vial que se presente se ven mas beneficiados unos departamentos y/o municipios que otros.

\section{Comparativo de los resultados de cobertura geoespacial}

La Figura 6a la cual representa una ojiva de población y la Figura 6b una ojiva del producto interno bruto (PIB) de la región, en las cuales se observa claramente que la alternativa No. 3 abarca mayor población y PIB de la región, teniendo en cuenta que presenta menores tiempos de viaje que las otras alternativas. Por ejemplo, tenemos que con un tiempo de viaje promedio de 10 horas se cubre actualmente el $82.21 \%$ de la población y un $81.78 \%$ del PIB de la región, mientras con la alternativa 1 se abarca el $85.11 \%$ de la población y el $84.77 \%$ del PIB, con la alternativa 2 se tiene el $85.23 \%$ de la población y el $84.54 \%$ del PIB, con la alternativa 3 se cubre el $86.37 \%$ de la población y el 85.64 del PIB, y finalmente con la alternativa de la APP se abarca el $82.59 \%$ de la población y el $82.30 \%$ del PIB. Concluyendo que desde este punto de vista la mejor alternativa es la No. 3.

Tabla 3. Departamentos y municipios con mejores porcentajes de ahorro. Fuente: Elaboración propia

\begin{tabular}{|c|c|c|c|c|c|c|c|c|}
\hline \multirow{2}{*}{ Alternativas } & \multicolumn{6}{|c|}{ Deptos y Municipios con Mejor Porcentaje de Ahorro o Mejor Gradiente de Tiempo Medio de Viaje } \\
\cline { 2 - 8 } & Depto & $\begin{array}{c}\text { \%Ahorro } \\
\text { Prom }\end{array}$ & Municipio & $\begin{array}{c}\text { \%Ahorro } \\
\text { Prom }\end{array}$ & $\begin{array}{c}\text { Municipio De } \\
\text { Caldas }\end{array}$ & $\begin{array}{c}\text { \%Ahorro } \\
\text { Prom }\end{array}$ & $\begin{array}{c}\text { Municipio De } \\
\text { La Region }\end{array}$ & $\begin{array}{c}\text { \%Ahorro } \\
\text { Prom }\end{array}$ \\
\hline $\begin{array}{c}\text { actual vs } \\
\text { ALT 1 }\end{array}$ & Caldas & 5,84 & Neira & 13,86 & Neira & 13,86 & Neira & 13,86 \\
\hline $\begin{array}{c}\text { actual vs } \\
\text { ALT 2 }\end{array}$ & Risaralda & 4,53 & Dosquebradas & 5,89 & Palestina & 5,89 & Casabianca & 11,94 \\
\hline $\begin{array}{c}\text { actual vs } \\
\text { ALT 3 }\end{array}$ & Risaralda & 5,74 & Dosquebradas & 7 & Neira & 12,25 & Neira & 12,25 \\
\hline $\begin{array}{c}\text { actual vs } \\
\text { ALT APP }\end{array}$ & Tolima & 1,49 & Libano & 13,47 & Villamaria & 1,64 & Libano & 13,47 \\
\hline
\end{tabular}

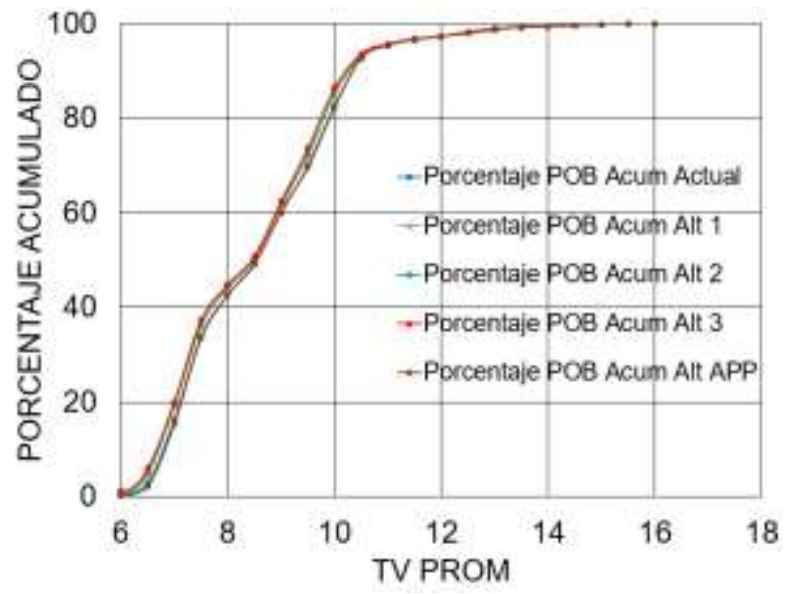

(a)

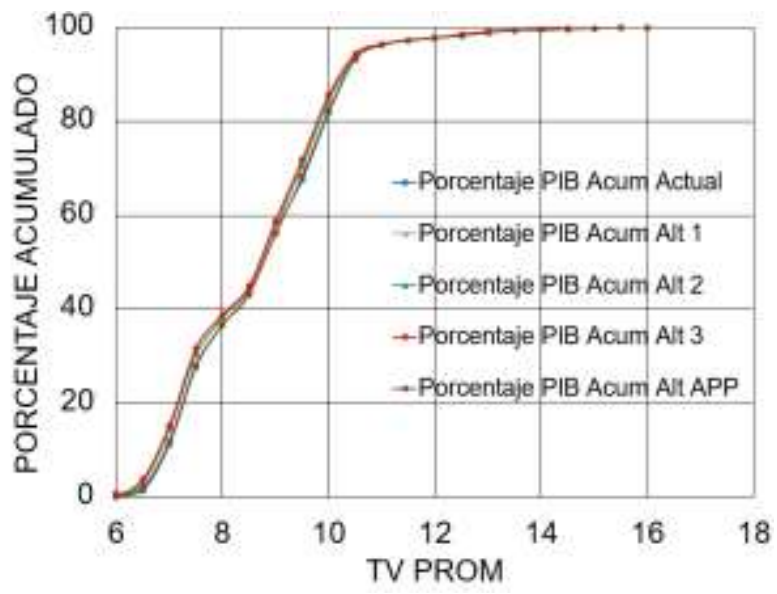

(b)

Fig. 6: Análisis acumulado de tiempo de viaje promedio en relación a la variable población y PIB del escenario actual y cada una de las alternativas. 


\section{Comparativo de los resultados de cobertura geoespacial}

Analizado la Figura 7a y la Figura $7 \mathrm{~b}$ se puede observar claramente como la alternativa No. 3 presenta mejores porcentajes de ahorro con relación a la población y PIB cubierta en comparación con las otras alternativas. Por ejemplo, tenemos que el $30 \%$ de la población para la alternativa de la APP presenta porcentajes de ahorro entre $0.8 \%$ y $25 \%$, que la alternativa 1 y 2 tienen porcentajes de ahorro entre $1.5 \%$ y $25 \%$, mientras que la alternativa No. 3 tiene porcentajes de ahorro entre $2.3 \%$ y $25 \%$. También tenemos que el $30 \%$ del Producto Interno Bruto (PIB) para la alternativa de la APP presenta porcentajes de ahorro entre $0.8 \%$ y $25 \%$, que la alternativa 1 y 2 tienen porcentajes de ahorro entre $1.3 \%$ y $25 \%$, mientras que la alternativa No. 3 tiene porcentajes de ahorro entre $2.1 \%$ y $25 \%$. Teniendo en cuenta el ejemplo mencionada seguimos observando como nuevamente la alternativa No. 3 se muestra como la más favorable.

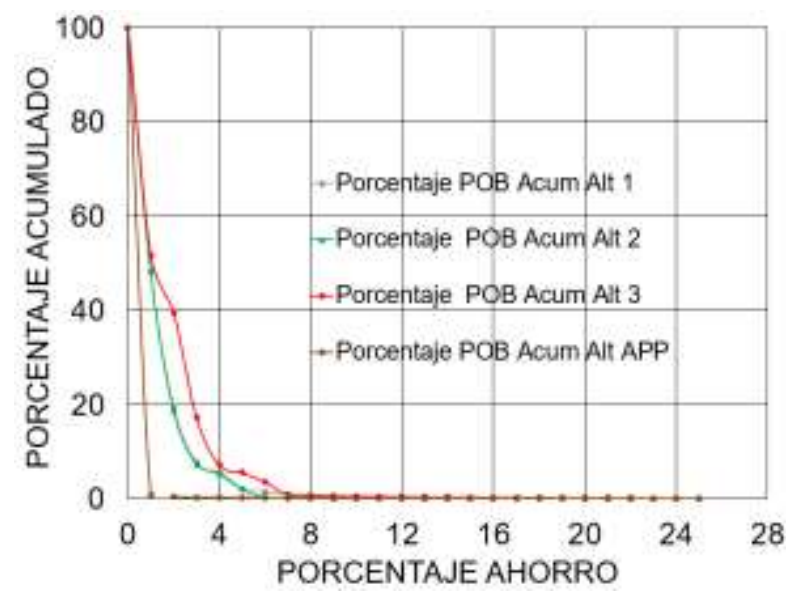

(a)

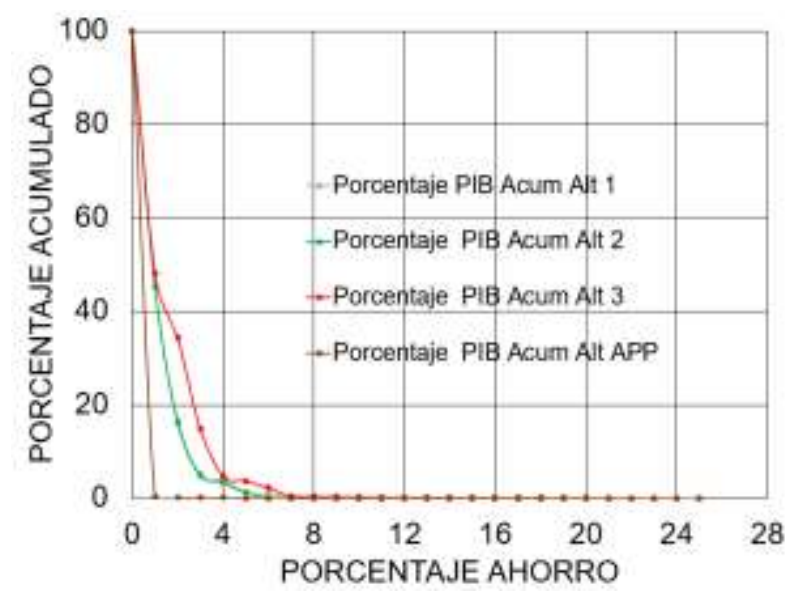

(b)

Fig. 7: Análisis acumulado de gradiente de tiempo de viaje promedio (porcentaje de ahorro) en relación a la variable población y PIB del escenario actual y cada una de las alternativas

\section{CONCLUSIONES}

Debido a la topografía que atraviesa la zona y los porcentajes de ahorro que se observaron en este estudio, se puede pensar en alternativas de transporte de pasajeros como el cable aéreo el cual es amigable para este tipo de zonas (Escobar et al., 2015), así como el mejorar la calidad del servicio de transporte público (Callejas-Cuervo et al., 2014) que actualmente circula entre dichos territorios, lo cual dependerá de una acertada selección de la infraestructura a construirse.

Al analizar la tabla 2 y 3 observamos como la alternativa 1 presenta mejores tiempos de viaje promedio y mayores porcentajes de ahorro para el departamento de Caldas, incluso mas que la alternativa 3, pero teniendo en cuenta que para seleccionar la mejor alternativa debemos poner en prelación la región en general más que a un departamento en específico, se vuelve viable la alternativa 3 debido a que es el trazado vial que abarca más población y mayor Producto Interno Bruto (PIB) de la región, es decir, es una solución que beneficia en un mayor pocentaje a los que integran la región analizada.

El gobierno nacional viene usando metodologías para la evaluación de proyectos de infraestructura vial totalmente diferentes a la aquí expuesta, sin embargo se ha demostrado que la alternativa seleccionada por el gobierno es la que menos beneficios representa para la comunidad, reflejando la necesidad de nuevos métodos para el análisis de este tipo de proyectos. Es claro que los beneficios en la región analizada se presentan en mayor proporción a nivel transversal que longitudinal, reflejándose en las pocas ganancias del Departamento de Antioquia y la buena relación de la infraestructura con el Departamento de Risaralda entendiendo que ninguna alternativa cruza dicho territorio, identificando a través de esta metodología la ausencia de conexión en el oriente de Caldas. Contextualizando esta conclusión con los ejemplos aplicados a nivel mundial, se observa cómo a partir de un análisis de accesibilidad territorial se conoce que población o que variable en particular impacta una infraestructura, además de tener el mismo panorama incluyendo diferentes proyectos en la región que se considere. Con el análisis de estas nuevas infraestructuras se puede tener un criterio, y teniendo en cuenta que se conoce cuál de ellas abarca en mayor proporción a la variable estudiada, se puede escoger una o varias alternativas para mejorar la accesibilidad de toda la región considerada con el mayor beneficio posible. 


\section{REFERENCIAS}

Agencia Nacional de Infraestructura, Listado de vías de cuarta generación (en la web: http://www.ani.gov.co/carreteras2, acceso: el 22 de junio de 2015), Ministerio de Transporte, Colombia (2014)

Agencia Nacional de Infraestructura, Seleccionar la Oferta más favorable para la Adjudicación de un Contrato de Concesión bajo el esquema de APP, cuyo objeto será La rehabilitación y mejoramiento de la vía Cambao-Manizales, y la rehabilitación del corredor vial Ibagué-Mariquita-Honda (en la web: https://www.contratos.gov.co/consultas/detalleProceso.do?numConstancia=15-19-3627936, acceso: 8 de abril de 2015), Sistema de Contratación Pública, Colombia (2015)

Alcaldía de Quibdó, Plan Integral de Movilidad de Quibdó 2015 - 2045, Universidad Nacional de Colombia Sede Manizales, Colombia (2015)

Biehl, D., The contribution of infrastructure to regional development. Final report of the Infrastructure Studies Group to the Commission of the European Communities, 1a edición, volume 2, Office for Official Publications of the European Communities, Luxembourg (1986)

Burns, C. M., y Inglis, A. D., Measuring food access in Melbourne: access to healthy and fast foods by car, bus and foot in an urban municipality in Melbourne, doi:10.1016/j.healthplace.2007.02.005, Health \& place (en línea), 13(4), 877-885 (2007)

Callejas-Cuervo, M., Valero-Bustos, H. y Alarcón-Aldana, A., Agentes de software como herramienta para medir la calidad de servicio prestado en un sistema de transporte público colectivo urbano, doi: 10.4067/S0718-07642014000500020, Inf. Tecnol., 25(5), 147-154 (2014)

Civiltec Ingenieros Ltda. (2013). Informe selección de alternativas, evaluación técnica,ambiental, socioeconomica y selección de alternativa, 1a edición, volumen 10, Instituto Nacional de Vias, Manizales, Colombia (2013)

Departamento Administrativo Nacional de Estadística, Censo General (en la web: http://www.dane.gov.co/index.php/poblacion-y-demografia/censos, acceso: 16 de abril de 2015), Colombia (2005)

Du, H., y Mulley, C., Relationship between transport accessibility and land value: Local model approach with geographically weighted regression, doi.org/10.3141/1977-25, Transportation Research Record: Journal of the Transportation Research Board (en línea), 1977, 197-205 (2006)

Duque, G., Frenado el transporte en Colombia (en la web:

http://www.unperiodico.unal.edu.co/dper/article/frenado-el-transporte-en-colombia.html, acceso 7 de abril de 2015), UN periódico, Colombia (2009)

Escobar D., García F. y Tolosa R., Análisis de Accesibilidad Territorial a Nivel Regional, 1aㅡ Edición, Universidad Nacional de Colombia. Facultad de Ingeniería y Arquitectura, Manizales, Colombia (2013).

Escobar, D. y García, F., Territorial Accessibility Analysis as a Key Variable for Diagnosis of Urban Mobility: A Case Study Manizales (Colombia), doi:10.1016/j.sbspro.2012.06.1114, Procedia - Social and Behavioral Sciences, 48 (0), pp. 1385-1394, (2012)

Escobar García, D.A.; Tapasco, O.A. y Giraldo, J.A., Medición de Desempeño del Sistema de Transporte Cable Aéreo de la Ciudad de Manizales en Colombia, usando Tres Enfoques: Analítico, Simulado y de Accesibilidad Urbana, doi: 10.4067/S0718-07642015000600020. Inf. Tecnol. [online]. 26(6), 199-210 (2015)

Geurs, K., Montis, A., \& Reggiani, A., Recent advances and applications in accessibility modelling, doi:10.1016/j.compenvurbsys.2014.09.003, (en línea), Computers, environment and urban systems, 49, 8285 (2015)

Geurs, K. T., Krizek, K. J., y Reggiani, A., Accessibility Analysis and Transport Planning, Challenges for Europe and North America, 1a edición, Twente, Holanda (2012)

Gutiérrez, J., Monzón, A., y Piñero, J. M., Accesibilidad a los centros de actividad económica en España, Revista de obras públicas, 141(3331), 34-49, España (1994) 
Gutiérrez, J., y Urbano, P. (1996). Accessibility in the European Union: the impact of the trans-European road network, doi:10.1016/0966-6923(95)00042-9, Journal of transport Geography (en línea),4(1), 15-25 (1996)

INVIAS, Instituto Nacional de Vias, Concurso de meritos: Estudios a nivel de fase I de la Conexión Manizales - Mariquita, (en la web:

https://www.contratos.gov.co/consultas/detalleProceso.do?numConstancia=11-10-122232, acceso 8 de abril de 2015), Sistema Electrónico de Contratación Pública, Colombia (2011)

Kotavaara, O., Antikainen, H. y Rusanen, J., Population change and accessibility by road and rail networks: GIS and statistical approach to Finland 1970-2007, doi:10.1016/j.jtrangeo.2010.10.013 Journal of Transport Geography, 19 (4), 926-935, (2011)

Li, Q., Zhang, T., Wang, H., \& Zeng, Z., Dynamic accessibility mapping using floating car data: A networkconstrained density estimation approach, doi:10.1016/j.jtrangeo.2010.07.003,Journal of Transport Geography (en línea), 19(3), 379-393 (2011)

Litman, T., Evaluating accessibility for transportation planning (en línea: http://www.vtpi.org/access.pdf, acceso 22 de junio de 2015), Victoria, BC: Victoria Transport Policy Institute, USA (2011)

López, E., Gutierrez, J. y Gómez, G., Measuring regional cohesion effects of large-scale transport infrastructure investment: an accessibility approach, doi: 10.1080/09654310701814629, European Planning Studies, 16 (2), 277-301, (2008)

Martínez, L., \& Viegas, J., Effects of transportation accessibility on residential property values: Hedonic Price Model in the Lisbon, Portugal, metropolitan area, doi.org/10.3141/2115-16 Transportation Research Record: Journal of the Transportation Research Board (en línea), 2115, 127-137 (2009)

Rietveld, P., \& Bruinsma, F., Is transport infrastructure effective?: transport infrastructure and accessibility: impacts on the space economy, 1a edición, Springer Science \& Business Media, Berlín, Germany (1998)

Sánchez, H. M., La accesibillidad regional y el efecto territorial de las infraestructuras de transporte. Aplicación en Castilla-La Mancha, Boletín de la Asociación de Geógrafos Españoles, (en la web: http://dialnet.unirioja.es/descarga/articulo/3938147/1.pdf, acceso 12 de abril de 2016), (59), 79-104 (2012)

Shen, G., Measuring Accessibility of Housing to Public-community Facilities Using Geographical Information Systems, doi: 10.1111/1467-940X.00056, Review of Urban \& Regional Development Studies 14(3), 235-255 (2002)

Venegas, F. R. y Rojas, R. I, Teoría y Práctica del Ordenamiento y Manejo Sustentable del Territorio: Tijuana-Rosarito-Tecate, Baja California, México, doi:10.1612/inf.tecnol.4077it.08, Inf. Tecnol., 20(3), 73-87 (2009)

Vickerman, R., Spiekermann, K., y Wegener, M., Accessibility and economic development in Europe, doi: 10.1080/00343409950118878, Regional studies (en línea), 33(1), 1-15 (1999)

Wang, Y., Monzon, A., y Di Ciommo, F., Assessing the accessibility impact of transport policy by a land-use and transport interaction model-The case of Madrid, doi:10.1016/j.compenvurbsys.2014.03.005 ,(en línea), Computers, Environment and Urban Systems, 49, 126-135 (2015) 